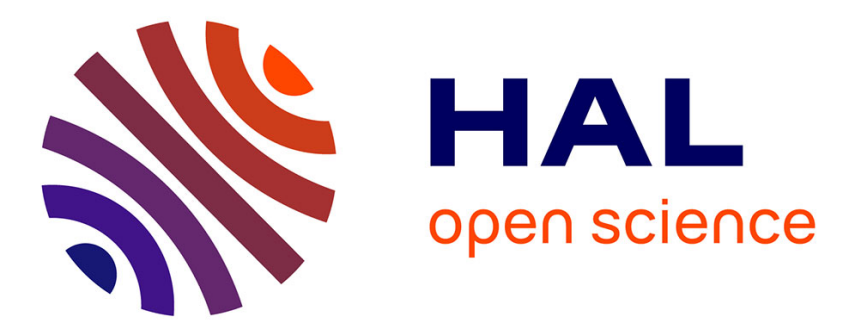

\title{
Analytical expressions for odd-order anisotropic tensor dimension
}

\author{
Nicolas Auffray
}

\section{To cite this version:}

Nicolas Auffray. Analytical expressions for odd-order anisotropic tensor dimension. Comptes Rendus Mécanique, 2014, 342 (5), pp.284-291. 10.1016/j.crme.2014.01.012 . hal-00922063

\section{HAL Id: hal-00922063 https://hal.science/hal-00922063}

Submitted on 23 Dec 2013

HAL is a multi-disciplinary open access archive for the deposit and dissemination of scientific research documents, whether they are published or not. The documents may come from teaching and research institutions in France or abroad, or from public or private research centers.
L'archive ouverte pluridisciplinaire HAL, est destinée au dépôt et à la diffusion de documents scientifiques de niveau recherche, publiés ou non, émanant des établissements d'enseignement et de recherche français ou étrangers, des laboratoires publics ou privés. 


\title{
Analytical expressions for odd-order anisotropic tensor dimension
}

\author{
N. Auffray ${ }^{a}$ \\ a MSME, Université Paris-Est, Laboratoire Modélisation et Simulation Multi Echelle, MSME UMR 8208 CNRS, 5 bd \\ Descartes, 77454 Marne-la-Vallée, France \\ Received $* * * * *$; accepted after revision +++++ \\ Presented by
}

\begin{abstract}
According to the symmetries of the matter the number of coefficients needed to define a tensorial relation varies. It is well-known that in linear elasticity the number of generic coefficients varies from 21 for a complete anisotropic material to 2 in case of isotropy. In a previous contribution, we provided analytical expressions that give the number of generic anisotropic coefficients in any anisotropic system for an even-order tensor. In the present note, we aim at extending the previous results to the case of odd-order tensors. As an illustration, the dimension of any anisotropic system for third-order piezoelectricity tensors and of the fifth-order coupling tensors of Mindlin's strain-gradient elasticity are determined. To cite this article: N. Auffray, C. R. Mécanique 341 (2013).
\end{abstract}

\section{Résumé}

Expressions analytiques donnant la dimension d'un tenseur anisotrope d'ordre impair. En fonction des symétries qu'un milieu possède, le nombre de coefficients génériques nécessaire à la définition d'une loi tensorielle varie. Dans le contexte de l'élasticité linéaire, si le milieu ne presente aucune symmétrie 21 coefficients élastiques sont génériquement nécessaire, tandis que dans le cas de l'isotropie ce nombre se réduit à 2 . Dans une précédente note, nous avions dérivé des formules analytiques donnant, dans le cas d'un tenseur pair, le nombre de coefficients génériques nécessaire pour chaque type d'anisotropie. Le but de cette nouvelle contribution, est de compléter ces formules en les étendant au cas des tenseurs impairs. En guise d'illustration, nous calculerons, pour l'ensemble des systèmes d'anisotropie possibles, la dimension des tenseurs piezoélectriques (ordre 3) ainsi que des tenseurs de couplage de la théorie de l'élasticité à gradient (ordre 5). Pour citer cet article : N. Auffray, C.R. Mécanique 341 (2013).

Key words: Anisotropic materials, Tensors, Generalized elasticity

Mots-clés : Matériaux anisotropes; Tenseurs; Elasticité généralisée

Email address: Nicolas.auffray@univ-mlv.fr (N. Auffray). 


\section{Introduction}

In the multiphysical modeling of anisotropic behaviors [1] it is interesting to have prior information on the general characteristics of the sought model. The knowledge, for a given material anisotropy, of the number of independent coefficients in the tensorial law is interesting since, for example, it determines the number of elementary tests one needs to perform in a micro-macro homogenization procedure [2]. These results are also important to develop strategies for the experimental identification of constitutive laws [4,3]. If in the case of classical elasticity these results are well-known [5,6], their extension to other situations is not straightforward. However, due to a growing interest in the modeling of size and non-local effects in materials and structures, the need for generalized continuum theories becomes evident $[7,8,9]$. This note, and following a path already developed in some previous contributions $[10,11,12]$, aims at providing some tools that can help the modeling of these non-conventional behaviors.

In the present paper, and following a previous one [12] in which only even-order tensors were considered, we provide analytical formulas that give the generic dimension of any anisotropic odd-order tensor ${ }^{1}$. To avoid any misunderstanding, it is worth noting that the present method does not solve the symmetry classification for a physical tensor, but only provide a way to compute, once the classification has been done, the number of generic coefficients for each anisotropic system. The solution of the classification problem can be found in the following references [25,23]. As an illustration, the obtained formula are applied to the space of third-order piezoelectric tensor [13,14], and to the fifth-order coupling tensor of Mindlin's strain-gradient elasticity [16].

This note is organized as follows, in Section 2 basic definitions about symmetries are summed up (see, e.g. [6] for an extended discussion). The decompositions of a tensor space into orthogonal elementary components and the notion of $G$-invariant space are developed in Section 3, and our main formula are provided there. Finally, physical illustrations are proposed in Section 4.

\section{Physical and material symmetries}

Hereafter $\mathrm{E}^{3}$ will be the 3D euclidean physical space. Let $G$ be a closed subgroup of $\mathrm{O}(3)$ the orthogonal group in 3D, that is the group of isometries of $\mathrm{E}^{3}$. Let us define a material $\mathcal{M}$ as an open subset of $\mathrm{E}^{3}$. The set of operations $\mathrm{Q} \in \mathrm{O}(3)$ leaving $\mathcal{M}$ invariant is defined as

$$
\mathrm{G}_{\mathcal{M}}=\{\mathrm{Q} \in \mathrm{O}(3), \quad \mathrm{Q} \star \mathcal{M}=\mathcal{M}\}
$$

where $\star$ stands for the $\mathrm{Q}$ action upon $\mathcal{M}$. This set, denoted $\mathrm{G}_{\mathcal{M}}$, is known as the material symmetry group. Now consider a physical property $\mathcal{P}$ defined on $\mathcal{M}$, the set of operations leaving $\mathcal{P}$ invariant is the physical symmetry group

$$
\mathrm{G}_{\mathcal{P}}=\{\mathrm{Q} \in \mathrm{O}(3), \quad \mathrm{Q} \star \mathcal{P}=\mathcal{P}\}
$$

$\mathcal{P}$ is described, in the present paper, by a $n^{\text {th }}$-order tensor $\mathrm{T}^{(n)} \in \mathbb{T}^{(n)}$. In that case the action $\star$ of $\mathrm{O}(3)$ on $T^{(n)}$ is defined by the Rayleigh product:

$$
\star: \mathrm{O}(3) \times \mathbb{T}^{(n)} \rightarrow \mathbb{T}^{(n)}:\left(\mathrm{Q}, \mathrm{T}^{(n)}\right) \mapsto \mathrm{Q} \star \mathrm{T}^{(n)}:=Q_{i_{1} j_{1}} \ldots Q_{i_{n} j_{n}} T_{j_{1} \ldots j_{n}}^{(n)}
$$

The material and the physical symmetry groups are related by the mean of the Curie-Neumann's principle [17]:

$$
\mathrm{G}_{\mathcal{M}} \subseteq \mathrm{G}_{\mathcal{P}}
$$

1. For a given group $G \in \mathrm{O}(3)$, by generic $G$-invariant tensors we mean tensors that only satisfy $G$-invariance and no other constraint. This is the case of almost all $G$-invariant tensors. 
meaning that each operation leaving the material invariant leaves the physical property invariant. Nevertheless, as shown for tensorial properties using Hermann's theorems [11], physical properties can be more symmetrical than the material.

In $\mathrm{E}^{3} \mathrm{G}_{\mathcal{P}}$ is conjugate to an $\mathrm{O}(3)$-closed subgroup [17,6]. Classification of $\mathrm{O}(3)$-closed subgroups is a classical result that can be found in many references, e.g. [18]:

Lemma 2.1 Every closed subgroup of $\mathrm{O}(3)$ is conjugate to precisely one group of the following list, which has been divided into three classes:

(i) Closed subgroups of $\mathrm{SO}(3): \mathbb{1}, \mathrm{Z}_{n}, \mathrm{D}_{n}, \mathcal{T}, \mathcal{O}, \mathcal{I}, \mathrm{SO}(2), \mathrm{O}(2), \mathrm{SO}(3)$;

(ii) $\tilde{K}:=K \oplus \mathrm{Z}_{2}^{c}$, where $K$ is a closed subgroup of $\mathrm{SO}(3)$ and $\mathrm{Z}_{2}^{c}=\{\mathbb{1},-\mathbb{1}\}$;

(iii) Closed subgroups not containing $-\mathbb{1}$ and not contained in $\mathrm{SO}(3)$ :

$$
\mathrm{Z}_{2 n}^{-}(n \geq 1), \mathrm{D}_{n}^{v}(n \geq 2), \mathrm{D}_{2 n}^{h}(n \geq 2), \mathcal{O}^{-} \text {or } \mathrm{O}(2)^{-}
$$

Let us now give a brief description of these different subgroups:

Type I subgroups

Among SO(3)-closed subgroups we can distinguish:

Planar groups : $\left\{\mathbb{1}, \mathrm{Z}_{n}, \mathrm{D}_{n}, \mathrm{SO}(2), \mathrm{O}(2)\right\}$, which are $\mathrm{O}(2)$-closed subgroups;

Exceptional groups : $\{\mathcal{T}, \mathcal{O}, \mathcal{I}, \mathrm{SO}(3)\}$, which are the rotation groups of chiral Platonic polyhedrons completed by the rotation group of the sphere.

Let us detail first the set of planar subgroups. We fix a base $(\mathbf{i} ; \mathbf{j} ; \mathbf{k})$ of $\mathbb{R}^{3}$, and denote by $\mathbf{Q}(\mathbf{v} ; \theta) \in \operatorname{SO}(3)$ the rotation about $\mathbf{v} \in \mathbb{R}^{3}$, with angle $\theta \in[0 ; 2 \pi)$ we have

- $\mathbb{1}$, the identity;

- $\mathrm{Z}_{n}(n \geq 2)$, the cyclic group of order $n$, generated by the $n$-fold rotation $\mathbf{Q}\left(\mathbf{k} ; \theta=\frac{2 \pi}{n}\right)$, which is the symmetry group of a chiral polygon;

- $\mathrm{D}_{n}(n \geq 2)$, the dihedral group of order $2 n$ generated by $\mathrm{Z}_{n}$ and $\mathbf{Q}(\mathbf{i} ; \pi)$, which is the symmetry group of a regular polygon;

- $\mathrm{SO}(2)$, the subgroup of rotations $\mathbf{Q}(\mathbf{k} ; \theta)$ with $\theta \in[0 ; 2 \pi)$. It is the symmetry group of an oriented cone.

- $\mathrm{O}(2)$, the subgroup generated by $\mathrm{SO}(2)$ and $\mathbf{Q}(\mathbf{i} ; \pi)$. It is the symmetry group of an twisted cylinder.

The classes of exceptional subgroups are: $\mathcal{T}$ the tetrahedral rotation group of order 12 which fixes a tetrahedron, $\mathcal{O}$ the octahedral rotation group of order 24 which fixes an octahedron (or a cube), and $\mathcal{I}$ the rotation group of order 60 which fixes an icosahedron (or a dodecahedron).

\section{Type II subgroups}

Type II subgroups are of the form $\tilde{K}:=K \oplus \mathrm{Z}_{2}^{c}$, where $K$ is a closed subgroup of $\mathrm{SO}(3)$. Therefore we directly know the collection of type II subgroups.

\section{Type III subgroups}

The construction of type III subgroups is more involved, and a description of their structure is provided in [18]. As for type I subgroups, we can introduce subgroups of type III. Let $\sigma_{\mathbf{u}} \in \mathrm{O}(3)$ denotes the reflection through the plane normal to $\mathbf{u}$ axis.

- $\mathrm{Z}_{2}^{-}$the order 2 reflection group generated by $\sigma_{\mathbf{i}}$; 
- $\mathrm{Z}_{2 n}^{-}(n \geq 2)$ the group of order $2 n$, generated by the $2 n$-fold rotoreflection $\mathbf{Q}\left(\mathbf{k} ; \theta=\frac{\pi}{n}\right) \cdot \sigma_{\mathbf{k}}$;

- $\mathrm{D}_{2 n}^{h}(n \geq 2)$ the prismatic group of order $4 n$ generated by $\mathrm{Z}_{2 n}^{-}$and $\mathbf{Q}(\mathbf{i}, \pi)$. When $n$ is odd it is the symmetry group of a regular prism, and when $n$ is even it is the symmetry group of a regular antiprism;

- $\mathrm{D}_{n}^{v}(n \geq 2)$ the pyramidal group of order $2 n$ generated by $\mathrm{Z}_{n}$ and $\sigma_{\mathbf{i}}$, which is the symmetry group of a regular pyramid;

- $\mathrm{O}(2)^{-}$is the limit group of $\mathrm{D}_{n}^{v}$ for continuous relation, it is therefore generated by $\mathbf{Q}(\mathbf{k} ; \theta)$ and $\sigma_{\mathbf{i}}$. It is the symmetry group of a cone;

- $\mathcal{O}^{-}$which is achiral tetrahedral symmetry of order 24. This group has the same rotation axes as $\mathcal{T}$, but with six mirror planes, each through two 3-fold axes.

In order to have a better physical understanding of these subgroups, we reported in Appendix A the tables making correspondences between group notations and the classical crystallographic ones (HermannMauguin, Schoenflies).

To study the symmetry classes of a tensor we need to decompose it into $\mathrm{O}(3)$-elementary parts.

\section{Structure of tensor spaces}

\subsection{Harmonic decomposition}

The $\mathrm{O}(3)$-invariant decomposition of a tensor is known as harmonic decomposition, it is an higherdimensional analogue of the Fourier decomposition. It allows to decompose any finite-order tensor into a sum of irreducible ones $[19,20]$. Formally, this decomposition can be written:

$$
\mathrm{T}^{n}=\sum_{k, \tau} \mathrm{D}(n)^{k, \tau}
$$

where tensors $\mathrm{D}(n)^{k, \tau}$ are components of the irreducible decomposition, $k$ denotes the order of the harmonic tensor embedded in $\mathrm{D}(n)$ and $\tau$ separates same order terms. This decomposition establishes an isomorphism between $\mathbb{T}^{n}$ and a direct sum of harmonic tensor spaces $\mathbb{H}^{k}[6]$ :

$$
\mathbb{T}^{n} \cong \bigoplus_{k, \tau} \mathbb{H}^{k, \tau}
$$

but, as explained, in [21] this decomposition may not be unique. Grouping together irreducible spaces of the same order, one obtains the $\mathrm{O}(3)$-isotypic decomposition of a representation which is unique [22]:

$$
\mathbb{T}^{n} \cong \bigoplus_{k=0}^{n} \alpha_{k} \mathbb{H}^{k}
$$

where $\alpha_{k}$ is the multiplicity of the irreducible space $\mathbb{H}^{k}$ in the decomposition and $n$ is the order of the highest-order irreducible space of the decomposition. $\mathbb{H}^{k}$ is the space of $k$ th-order harmonic tensors, that is the space of totally symmetric, traceless tensors of order $k$. It is a vector space of dimension $2 k+1$ in $\mathbb{R}^{3}$. The series $\left\{\alpha_{k}\right\}$ is function of the order and the intrinsic symmetries of the tensor space. Various methods exist to compute this family $[20,19,10]$. 
Before closing this rather short introduction, it is important to note that in the decomposition of an even-order (resp. odd-order) tensor, odd-order (resp. even order) components are pseudo-tensors ${ }^{2}$, i.e change sign if the space orientation is reversed.

\subsection{Dimension of $G$-invariant harmonic spaces}

Let $G$ be any subgroup of $\mathrm{O}(3)$. The set of tensors $\mathrm{T} \in \mathbb{T}$ which are fixed by $G$

$$
\mathbb{F i x}_{\mathbb{T}}(G):=\{\mathrm{T} \in \mathbb{T} \mid g . \mathrm{T}=\mathrm{T} \text { for all } g \in G\},
$$

is called the fixed point set ${ }^{3}$. It is the biggest linear subspace of $\mathbb{T}$ that contains $G$-invariant tensors. Elements of $\mathbb{F i x}_{\mathbb{T}}(G)$ only defined in terms of $G$-invariance without any further constraint will be referred to as generic. Since non-generic elements constitute a null set they will not be considered here.

It is worth noting that the dimension of type II invariant subspaces is always 0. And for O(3)-subgroups of types I and III we have the following results ${ }^{4}$ concerning harmonic tensor spaces.

\section{Type I subgroups ${ }^{5}$}

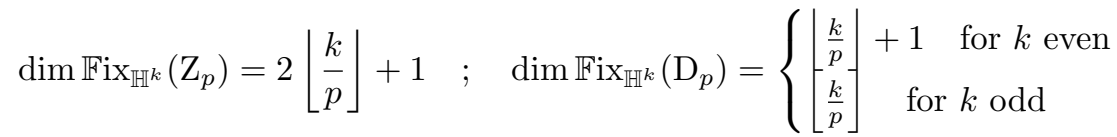

$$
\begin{aligned}
& \operatorname{dim} \mathbb{F i x}_{\mathbb{H}^{k}}(\mathcal{T})=2\left\lfloor\frac{k}{3}\right\rfloor+\left\lfloor\frac{k}{2}\right\rfloor-k+1 \quad ; \quad \operatorname{dim} \mathbb{F i x}_{\mathbb{H}^{k}}(\mathcal{O})=\left\lfloor\frac{k}{4}\right\rfloor+\left\lfloor\frac{k}{3}\right\rfloor+\left\lfloor\frac{k}{2}\right\rfloor-k+1 \\
& \operatorname{dim} \operatorname{Fix}_{\mathbb{H}^{k}}(\mathcal{I})=\left\lfloor\frac{k}{5}\right\rfloor+\left\lfloor\frac{k}{3}\right\rfloor+\left\lfloor\frac{k}{2}\right\rfloor-k+1
\end{aligned}
$$

Type III subgroups

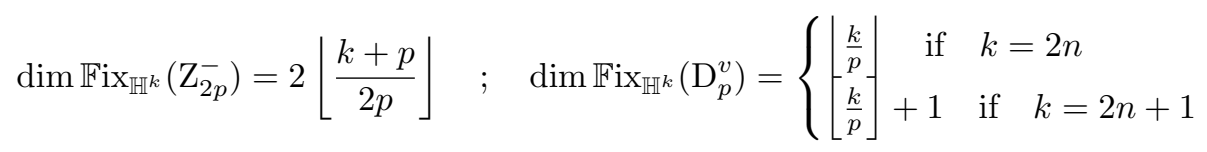

$$
\begin{aligned}
& \operatorname{dim} \mathbb{F i x}_{\mathbb{H}^{k}}\left(\mathrm{D}_{2 p}^{h}\right)=\left\lfloor\frac{k+p}{2 p}\right\rfloor \quad ; \quad \operatorname{dim}_{\mathbb{F i x}} \mathbb{H}_{\mathbb{H}^{k}}\left(\mathrm{SO}(2)^{-}\right)= \begin{cases}0 & k=2 n \\
1 & k=2 n+1\end{cases} \\
& \operatorname{dim} \mathbb{F i x}_{\mathbb{H}^{k}}\left(\mathcal{O}^{-}\right)=\left\lfloor\frac{k}{3}\right\rfloor-\left\lfloor\frac{k}{4}\right\rfloor
\end{aligned}
$$

These elementary results, combined with the knowledge of the isotypic decomposition of a tensor space (3.1), allow to determine the dimension of any $G$-invariant tensor subspaces. Analytical expressions are constructed according to that procedure.

\footnotetext{
2. The related tensors are multiplied by the Levi-Civita symbol $\epsilon_{i j k}$.

3. As $G$ as a action on the space $\mathbb{T}$, there is an homeomorphism $\psi$ from $G$ to $\operatorname{GL}(\mathbb{T})$. Hence the notation $g$. T should be understand as a classical shortcut to the more rigorous one $\psi(g)$. T.

4. More details can be found in $[21,12]$.

5. The formulas for type I subgroup were already provided in [12]. They are summed-up here for the sake of completeness.
} 


\subsection{G-invariant tensor subspaces}

Applying this process the following formulas are obtained:

\section{Type I subgroups}

\section{- $\mathrm{Z}_{p}$-invariance}

$$
\operatorname{dim} \mathbb{F i x}_{\mathbb{T}}\left(\mathrm{Z}_{p}\right)=2 \sum_{k=0}^{n} \alpha_{k}\left\lfloor\frac{k}{p}\right\rfloor+\sum_{k=0}^{n} \alpha_{k}
$$

When $p>k$ we obtain $\left\lfloor\frac{k}{p}\right\rfloor=0$ and so $\beta_{\text {oth }}=\sum_{k=0}^{n} \alpha_{k}$ is the number of oriented transverse hemitropic coefficients. $\beta_{\text {oth }}$ is the dimension of a $\mathrm{SO}(2)$-invariant tensor.

$\bullet \mathrm{D}_{p}$-invariance

$$
\operatorname{dim} \operatorname{Fix}_{\mathbb{T}}\left(\mathrm{D}_{p}\right)=\sum_{k=0}^{n} \alpha_{k}\left\lfloor\frac{k}{p}\right\rfloor+\sum_{k=0}^{\left\lfloor\frac{n}{2}\right\rfloor} \alpha_{2 k}
$$

When $p>k$ we obtain $\left\lfloor\frac{k}{p}\right\rfloor=0$ and so $\beta_{t t i}=\sum_{k=0}^{n} \alpha_{k}$ is the number of twisted transverse isotropic coefficients. $\beta_{t t i}$ is the dimension of an $\mathrm{O}(2)$-invariant tensor.

- $\mathcal{T}, \mathcal{O}$ and $\mathcal{I}$-invariance

$$
\begin{gathered}
\operatorname{dim} \operatorname{Fix}_{\mathbb{T}}(\mathcal{T})=\sum_{k=0}^{n} \alpha_{k}\left(2\left\lfloor\frac{k}{3}\right\rfloor+\left\lfloor\frac{k}{2}\right\rfloor-k+1\right) ; \operatorname{dim} \mathbb{F i x}_{\mathbb{T}}(\mathcal{O})=\sum_{k=0}^{n} \alpha_{k}\left(\left\lfloor\frac{k}{4}\right\rfloor+\left\lfloor\frac{k}{3}\right\rfloor+\left\lfloor\frac{k}{2}\right\rfloor-k+1\right) \\
\operatorname{dim} \mathbb{F i x}_{\mathbb{T}}(\mathcal{I})=\sum_{k=0}^{n} \alpha_{k}\left(\left\lfloor\frac{k}{5}\right\rfloor+\left\lfloor\frac{k}{3}\right\rfloor+\left\lfloor\frac{k}{2}\right\rfloor-k+1\right)
\end{gathered}
$$

Type III subgroups

- $\mathrm{Z}_{2 p}^{-}$-invariance

$$
\operatorname{dim} \mathbb{F i x}_{\mathbb{T}}\left(\mathrm{Z}_{2 p}^{-}\right)=2 \sum_{k=0}^{n} \alpha_{k}\left\lfloor\frac{k+p}{2 p}\right\rfloor
$$

When $p>k$ we obtain $\left\lfloor\frac{k+p}{2 p}\right\rfloor=0$ and therefore the tensor is null. In such a case, the tensor is obviously $\mathrm{O}(3)$-invariant.

- $\mathrm{D}_{2 p}^{h}$-invariance

$$
\operatorname{dim} \mathbb{F i x}_{\mathbb{T}}\left(\mathrm{D}_{2 p}^{h}\right)=\sum_{k=0}^{n} \alpha_{k}\left\lfloor\frac{k+p}{2 p}\right\rfloor=\frac{1}{2} \operatorname{dim} \mathbb{F i x}_{\mathbb{T}}\left(\mathrm{Z}_{2 p}^{-}\right)
$$

When $p>k$ we obtain $\left\lfloor\frac{k+p}{2 p}\right\rfloor=0$ and therefore the tensor is null. In such a case, the tensor is obviously $\mathrm{O}(3)$-invariant. 
- $\mathrm{D}_{p}^{v}$-invariance

$$
\operatorname{dim} \operatorname{Fix}_{\mathbb{T}}\left(\mathrm{D}_{p}^{v}\right)=\sum_{k=0}^{n} \alpha_{k}\left\lfloor\frac{k}{p}\right\rfloor+\sum_{k=0}^{\left\lfloor\frac{n-1}{2}\right\rfloor} \alpha_{2 k+1}
$$

When $p>k$ we obtain $\left\lfloor\frac{k}{p}\right\rfloor=0$ and so $\beta_{t h}=\sum_{k=0}^{\left\lfloor\frac{n-1}{2}\right\rfloor} \alpha_{2 k+1}$ is the number of non-oriented transverse hemitropic coefficients. $\beta_{t h}$ is the dimension of an $\mathrm{O}(2)^{-}$-invariant tensor.

\section{- $\mathcal{O}^{-}$-invariance}

$$
\operatorname{dim} \mathbb{F i x}_{\mathbb{T}}\left(\mathcal{O}^{-}\right)=\sum_{k=0}^{n} \alpha_{k}\left(\left\lfloor\frac{k}{3}\right\rfloor-\left\lfloor\frac{k}{4}\right\rfloor\right)
$$

\section{Physical results}

In order to illustrate the practical interest of these formulas two examples will be considered: the thirdorder tensor of piezoelectricity and the fifth-order coupling tensor of Mindlin's strain gradient elasticity.

\subsection{Piezoelectricity}

Let us consider Piez the vector space of piezoelectricity tensors, its elements are symmetric under the permutation of their two first indices: $\mathrm{P}_{(i j) k}$, where $(.$.$) stands for the minor symmetry. It has been shown$ $[13,14]$ that this vector space is isomorphic to:

$$
\mathbb{P i e z} \cong 2 \mathbb{H}^{1} \oplus \mathbb{H}^{\sharp 2} \oplus \mathbb{H}^{3}
$$

where the ${ }^{\sharp}$ notation indicates a pseudo-tensor. And so Piez is defined by the following $\left\{\alpha_{k}\right\}$ family: $\{0,2,1,1\}$. As determined in $[13,14,23]$ the space of piezoelectric tensors can be divided into the following anisotropic systems:

$$
[\mathbb{P i e z}]=\left\{[\mathbb{1}],\left[\mathrm{Z}_{2}\right],\left[\mathrm{Z}_{3}\right],\left[\mathrm{D}_{2}^{v}\right],\left[\mathrm{D}_{3}^{v}\right],\left[\mathrm{Z}_{2}^{-}\right],\left[\mathrm{Z}_{4}^{-}\right],\left[\mathrm{D}_{2}\right],\left[\mathrm{D}_{3}\right],\left[\mathrm{D}_{4}^{h}\right],\left[\mathrm{D}_{6}^{h}\right],[\mathrm{SO}(2)],[\mathrm{O}(2)],\left[\mathrm{O}(2)^{-}\right],\left[\mathcal{O}^{-}\right]\right\}
$$

Straightforward applications of our formula give

\section{-Type I subgroups}

\begin{tabular}{|c||c|c|c|c||c|c|c|}
\hline$[\mathbb{P i e z}]$ & {$[\mathbb{1}]$} & {$\left[\mathrm{Z}_{2}\right]$} & {$\left[\mathrm{Z}_{3}\right]$} & {$[\mathrm{SO}(2)]$} & {$\left[\mathrm{D}_{2}\right]$} & {$\left[\mathrm{D}_{3}\right]$} & {$[\mathrm{O}(2)]$} \\
\hline $\operatorname{dim}$ & 18 & 8 & 6 & 4 & 3 & 2 & 1 \\
\hline
\end{tabular}

\section{-Type III subgroups}

\begin{tabular}{|c||c|c||c|c|c||c|c||c|}
\hline$[$ Piez $]$ & {$\left[\mathrm{Z}_{2}^{-}\right]$} & {$\left[\mathrm{Z}_{4}^{-}\right]$} & {$\left[\mathrm{D}_{2}^{v}\right]$} & {$\left[\mathrm{D}_{3}^{v}\right]$} & {$\left[\mathrm{O}^{-}(2)\right]$} & {$\left[\mathrm{D}_{4}^{h}\right]$} & {$\left[\mathrm{D}_{6}^{h}\right]$} & {$\left[\mathcal{O}^{-}\right]$} \\
\hline $\operatorname{dim}$ & 10 & 4 & 5 & 4 & 3 & 2 & 1 & 1 \\
\hline
\end{tabular}

where the number of coefficients for each physical symmetry class have been determined ${ }^{6}$. These results are obviously in agreement with the ones in the literature $[13,14,15]$.

6. A precise definition of symmetry classes can be found in the following references $[6,25,23]$. 


\subsection{Mindlin strain-gradient elasticity}

Let us consider $\mathbb{E} l \mathrm{a}_{\mathrm{M}}$ the vector space of the coupling tensors in Mindlin strain gradient elasticity. It is the vector space of fifth-order tensors endowed with the following index symmetries [16]: $\mathrm{M}_{(i j)(k l) m}$. It has been shown [11] that this vector space decomposed as follows :

$$
\mathbb{E} \mathrm{la}_{\mathrm{M}} \cong \mathbb{H}^{\sharp 0} \oplus 6 \mathbb{H}^{1} \oplus 5 \mathbb{H}^{\sharp 2} \oplus 5 \mathbb{H}^{3} \oplus 2 \mathbb{H}^{\sharp 4} \oplus \mathbb{H}^{5}
$$

and so $\mathbb{E}$ la $\mathrm{M}_{\mathrm{M}}$ is defined by the following $\left\{\alpha_{k}\right\}$ family: $\{1,6,5,5,2,1\}$. As determined in [23] the space $\mathbb{E}$ la $\mathrm{M}$ can be divided into the following anisotropic systems:

$$
\begin{aligned}
{[\mathbb{E l a} \mathrm{M}]=} & \left\{[\mathbb{1}],\left[\mathrm{Z}_{2}\right], \cdots,\left[\mathrm{Z}_{5}\right],\left[\mathrm{D}_{2}^{v}\right], \cdots,\left[\mathrm{D}_{5}^{v}\right],\left[\mathrm{Z}_{2}^{-}\right], \cdots,\left[\mathrm{Z}_{8}^{-}\right],\left[\mathrm{D}_{2}\right], \cdots,\left[\mathrm{D}_{5}\right]\right. \\
& {\left.\left[\mathrm{D}_{4}^{h}\right], \cdots,\left[\mathrm{D}_{10}^{h}\right],[\mathrm{SO}(2)],[\mathrm{O}(2)],\left[\mathrm{O}(2)^{-}\right],[\mathcal{T}],\left[\mathcal{O}^{-}\right],[\mathcal{O}],[\mathrm{SO}(3)],[\mathrm{O}(3)]\right\} }
\end{aligned}
$$

Therefore $\mathbb{E}_{\mathrm{a}} \mathrm{a}$ is divided into 28 symmetry classes ${ }^{7}$. This large number of symmetry classes has to be compared with the 8 symmetry classes of classical elasticity [6], and the 17 symmetry classes of second order elasticity $[24,25]$. Straightforward applications of our formula now give

\section{-Type I subgroups}

\begin{tabular}{|c||c|c|c|c|c|c||c|c|c|c|c||c|c|c|}
\hline$\left[\mathbb{E} l_{\mathrm{M}}\right]$ & {$[\mathbb{1}]$} & {$\left[\mathrm{Z}_{2}\right]$} & {$\left[\mathrm{Z}_{3}\right]$} & {$\left[\mathrm{Z}_{4}\right]$} & {$\left[\mathrm{Z}_{5}\right]$} & {$[\mathrm{SO}(2)]$} & {$\left[\mathrm{D}_{2}\right]$} & {$\left[\mathrm{D}_{3}\right]$} & {$\left[\mathrm{D}_{4}\right]$} & {$\left[\mathrm{D}_{5}\right]$} & {$[\mathrm{O}(2)]$} & {$[\mathcal{T}]$} & {$[\mathcal{O}]$} & {$[\mathrm{SO}(3)]$} \\
\hline $\operatorname{dim}$ & 108 & 52 & 36 & 26 & 22 & 20 & 24 & 16 & 11 & 9 & 8 & 8 & 3 & 1 \\
\hline
\end{tabular}

\section{•Type III subgroups}

\begin{tabular}{|c||c|c|c|c||c|c|c|c|c||c|c|c|c||c|}
\hline$\left[\mathbb{E} \mathrm{a}_{\mathrm{M}}\right]$ & {$\left[\mathrm{Z}_{2}^{-}\right]$} & {$\left[\mathrm{Z}_{4}^{-}\right]$} & {$\left[\mathrm{Z}_{6}^{-}\right]$} & {$\left[\mathrm{Z}_{8}^{-}\right]$} & {$\left[\mathrm{D}_{2}^{v}\right]$} & {$\left[\mathrm{D}_{3}^{v}\right]$} & {$\left[\mathrm{D}_{4}^{v}\right]$} & {$\left[\mathrm{D}_{5}^{v}\right]$} & {$\left[\mathrm{O}^{-}(2)\right]$} & {$\left[\mathrm{D}_{4}^{h}\right]$} & {$\left[\mathrm{D}_{6}^{h}\right]$} & {$\left[\mathrm{D}_{8}^{h}\right]$} & {$\left[\mathrm{D}_{10}^{h}\right]$} & {$\left[\mathcal{O}^{-}\right]$} \\
\hline $\operatorname{dim}$ & 56 & 26 & 16 & 6 & 28 & 20 & 15 & 13 & 12 & 13 & 8 & 3 & 1 & 5 \\
\hline
\end{tabular}

\section{Conclusion}

In this note, analytical formulas giving the dimension of a subspace left fixed under $\mathrm{O}(3)$-subgroups action have been provided and applied to the symmetry classes of some physical tensor spaces. To be applied, the only things that has to be known are the $\mathrm{O}(3)$-isotypic decomposition of the studied tensor space, and the symmetry classes of the tensors spaces. Using the method proposed in $[20,11]$, this decomposition is easily obtained, and the determination of the symmetry classes is completely solved in $[?, 23]$. We believe that these simple formulas can be of great help to develop, for example, higher-order constitutive laws $[26,8]$ and to design micro-macro homogenization procedure for anisotropic materials $[2]$.

7. Or 29, if the class $[\mathrm{O}(3)]$ of null tensors is taken into account. But this point is only a question of convention 
Appendix A. Dictionary between group notations and crystallographic systems $\mathrm{O}(3)$ type I closed-subgroups

\begin{tabular}{|c|c|c|}
\hline Hermann-Maugin & Schonflies & Group \\
\hline 1 & $\mathrm{C}_{1}$ & $\mathbb{1}$ \\
\hline 2 & $\mathrm{C}_{2}$ & $\mathrm{Z}_{2}$ \\
\hline 222 & $\mathrm{D}_{2}$ & $\mathrm{D}_{2}$ \\
\hline 3 & $\mathrm{C}_{3}$ & $\mathrm{Z}_{3}$ \\
\hline 32 & $\mathrm{D}_{3}$ & $\mathrm{D}_{3}$ \\
\hline 4 & $\mathrm{C}_{4}$ & $\mathrm{Z}_{4}$ \\
\hline 422 & $\mathrm{D}_{4}$ & $\mathrm{D}_{4}$ \\
\hline 6 & $\mathrm{C}_{6}$ & $\mathrm{Z}_{6}$ \\
\hline 622 & $\mathrm{D}_{6}$ & $\mathrm{D}_{6}$ \\
\hline$\infty$ & $\mathrm{C}_{\infty}$ & $\mathrm{SO}(2)$ \\
\hline$\infty 2$ & $\mathrm{D}_{\infty}$ & $\mathrm{O}(2)$ \\
\hline 23 & $\mathrm{~T}$ & $\mathcal{T}$ \\
\hline 432 & $\mathrm{O}$ & $\mathcal{O}$ \\
\hline 532 & $\mathrm{I}$ & $\mathcal{I}$ \\
\hline$\infty \infty$ & & $\mathrm{SO}(3)$ \\
\hline & &
\end{tabular}


$\mathrm{O}(3)$ type II closed-subgroups

\begin{tabular}{|c|c|c|}
\hline Hermann-Maugin & Schonflies & Group \\
\hline$\overline{1}$ & $\mathrm{C}_{i}$ & $\mathrm{Z}_{2}^{c}$ \\
\hline $2 / m$ & $\mathrm{C}_{2 h}$ & $\mathrm{Z}_{2} \oplus \mathrm{Z}_{2}^{c}$ \\
\hline$m m m$ & $\mathrm{D}_{2 h}$ & $\mathrm{D}_{2} \oplus \mathrm{Z}_{2}^{c}$ \\
\hline$\overline{3}$ & $\mathrm{~S}_{6}, \mathrm{C}_{3 i}$ & $\mathrm{Z}_{3} \oplus \mathrm{Z}_{2}^{c}$ \\
\hline$\overline{3} m$ & $\mathrm{D}_{3 d}$ & $\mathrm{D}_{3} \oplus \mathrm{Z}_{2}^{c}$ \\
\hline $4 / m$ & $\mathrm{C}_{4 h}$ & $\mathrm{Z}_{4} \oplus \mathrm{Z}_{2}^{c}$ \\
\hline $4 / m m m$ & $\mathrm{D}_{4 h}$ & $\mathrm{D}_{4} \oplus \mathrm{Z}_{2}^{c}$ \\
\hline $6 / m$ & $\mathrm{C}_{6 h}$ & $\mathrm{Z}_{6} \oplus \mathrm{Z}_{2}^{c}$ \\
\hline $6 / m m m$ & $\mathrm{D}_{6 h}$ & $\mathrm{D}_{6} \oplus \mathrm{Z}_{2}^{c}$ \\
\hline$m \overline{3}$ & $\mathrm{~T}_{h}$ & $\mathcal{T} \oplus \mathrm{Z}_{2}^{c}$ \\
\hline$m \overline{3} m$ & $\mathrm{O}_{h}$ & $\mathcal{O} \oplus \mathrm{Z}_{2}^{c}$ \\
\hline$\overline{5} \overline{3} m$ & $\mathrm{I}_{h}$ & $\mathcal{I} \oplus \mathrm{Z}_{2}^{c}$ \\
\hline$\infty / m$ & $\mathrm{C}_{\infty h}$ & $\mathrm{SO}(2) \oplus \mathrm{Z}_{2}^{c}$ \\
\hline$\infty / m m$ & $\mathrm{D}_{\infty h}$ & $\mathrm{O}(2) \oplus \mathrm{Z}_{2}^{c}$ \\
\hline$\infty / m \infty / m$ & & $\mathrm{O}(3)$ \\
\hline
\end{tabular}




\begin{tabular}{|c|c|c|}
\hline Hermann-Maugin & Schonflies & Group \\
\hline$m$ & $\mathrm{C}_{s}$ & $\mathrm{Z}_{2}^{-}$ \\
\hline $2 m m$ & $\mathrm{C}_{2 v}$ & $\mathrm{D}_{2}^{v}$ \\
\hline $3 m$ & $\mathrm{C}_{3 v}$ & $\mathrm{D}_{3}^{v}$ \\
\hline$\overline{4}$ & $\mathrm{~S}_{4}$ & $\mathrm{Z}_{4}^{-}$ \\
\hline $4 m m$ & $\mathrm{C}_{4 v}$ & $\mathrm{D}_{4}^{v}$ \\
\hline$\overline{4} 2 m$ & $\mathrm{D}_{2 d}$ & $\mathrm{D}_{4}^{h}$ \\
\hline$\overline{6}$ & $\mathrm{C}_{3 h}$ & $\mathrm{Z}_{6}^{-}$ \\
\hline $6 m m$ & $\mathrm{C}_{6 v}$ & $\mathrm{D}_{6}^{v}$ \\
\hline$\overline{6} 2 m$ & $\mathrm{D}_{3 h}$ & $\mathrm{D}_{6}^{h}$ \\
\hline$\overline{4} 3 m$ & $\mathrm{~T}_{d}$ & $\mathcal{O}^{-}$ \\
\hline$\infty m$ & $\mathrm{C}_{\infty v}$ & $\mathrm{O}_{(2}$ \\
\hline
\end{tabular}

\section{References}

[1] A. Thionnet, C. Martin, A new constructive method using the theory of invariants to obtain material behavior laws, Int. J. Solids Struct. 43 (2006) 325-345.

[2] D.-K. Trinh, R. Jänicke, N. Auffray, S. Diebels, S. Forest, Evaluation of generalized continuum substitution models for heterogeneous materials. Int. J. Multiscale. Com., 10, (2012) 527-549.

[3] M., Francois, G. Geymonat, Y. Berthaud. Determination of the symmetries of an experimentally determined stiffness tensor: application to acoustic measurements. Int. J. Solids Struct. 35 (1998) 4091-4106.

[4] M., Francois, Y. Berthaud, G. Geymonat. Une nouvelle analyse des symétries d'un matéeriau élastique anisotrope. Exemple d'utilisation à partir de mesures ultrasonores. C.R. Mecanique -Series IIB, 322 (1996) 87-94.

[5] S. C. Cowin, M. M. Mehrabadi, Eigentensors of linear anisotropic elastic materials. Q. J. Mech. Appl. Math., 43 (1990) $15-41$.

[6] S. Forte, M. Vianello, Symmetry classes for elasticity tensors, J. Elasticity 43 (1996) 81-108.

[7] N.A. Fleck, J.W. Hutchinson, Strain gradient plasticity, J.W. Hutchinson, T.Y. Wu (Eds.), Advances in Applied Mechanics, vol.33 Academic Press, New York 1997.

[8] J. Alibert, P. Seppecher, F. dell'Isola, Truss modular beams with deformation energy depending on higher displacement gradients, Math. Mech. Solids., 8 (2003) 51-73.

[9] G. Sciarra, F. dell'Isola, O. Coussy, Second gradient poromechanics, Int. J. Solids Struct. 44 (2007) 6607-6629.

[10] N. Auffray, Démonstration du théoréme d'Hermann é partir de la méthode Forte-Vianello, C.R. Mecanique 336 (2008) 458-463.

[11] N. Auffray, Décomposition harmonique des tenseurs -Méthode spectrale-, C.R. Mecanique 336 (2008) 370-375.

[12] N. Auffray, Analytical expressions for anisotropic tensor dimension, C.R. Mecanique 338 (2010) $260-265$.

[13] T. Weller, Etude des symétries et modèles de plaques en piézoélectricité linéarisée, PhD Thesis, Université Montpellier II, 2004. 
[14] T. Weller, G, Geymonat, Piezomagnetic tensors symmetries: an unifying tentative approach. In Configurational Mechanics: Proceedings of the Configurational Mechanics, 2004, Taylor \& Francis.

[15] W. N. Zou, C. X. Tang, E. Pan, Symmetry types of the piezoelectric tensor and their identification. Proc. R. Soc. A $469(2013)$

[16] R.D. Mindlin, N.N. Eshel, On first strain-gradient theories in linear elasticity, Int. J. Solids Struct. 4 (1968) $109-124$.

[17] Q.-S. Zheng, J. P. Boehler, The description, classification, and reality of material and physical symmetries. Acta. Mech. 102(1994) 73-89.

[18] S. Sternberg, Group theory and physics. Cambridge: Cambridge University Press (1994).

[19] W. Zou, Q.-S. Zheng, D. Du, J. Rychlewski, Orthogonal Irreducible Decompositions of Tensors of High Orders, Math. Mech. Solids. , 2001, 6, 249-26

[20] J. Jerphagnon, D. Chemla, R. Bonneville, The description of the physical properties of condensed matter using irreducible tensors, Adv. Phys. 27 (1978) 609-650.

[21] M. Golubitsky, I. Stewart, D. Schaeffer, Singularities and Groups in Bifurcation Theory. Volume II. Springer-Verlag (1989)

[22] Y. Kosmann-Schwarzbach, Groupes et symétries. Groupes finis, groupes et algébres de Lie, représentations, Edition Ecole Polytechnique, 2005.

[23] M. Olive, N. Auffray, Symmetry classes for odd-order tensors, Z. Angew. Math. Mech. (under revision).

[24] N. Auffray, H. Le Quang, Q.-C. He, Matrix representations for 3D strain-gradient elasticity, J. Mech. Phys. Solids 61 (2013) 1202-1223.

[25] M. Olive, N. Auffray, Symmetry classes for even-order tensors, Mathematics and Mechanics of Complex Systems 1, accepted (2013).

[26] F. dell'Isola, P. Sciarra, S. Vidoli, Generalized Hooke's law for isotropic second gradient materials, Proc. R. Soc. A 465 (2009) 2177-2196. 